\title{
A LOGLINEAR LAGRANGIAN POISSON MODEL
}

\author{
By Peter ter Berg
}

SFB Verzekeringen, Amsterdam, Netherlands

\begin{abstract}
Maximum likelihood estimation is derived for the Lagrangian Poisson distribution for a simple and a loglinear model and illustrated with real data.
\end{abstract}

\section{KEYWORDS}

Loglinear; Lagrangian Poisson; Maximum likelihood; Newton-Raphson.

\section{INTRODUCTION}

The monograph by Consul (1989) is on a nice counting distribution known as Lagrangian Poisson'

$$
\begin{gathered}
p(n \mid \theta, \zeta)=\theta(\theta+n \zeta)^{n-1} \exp [-(\theta+n \zeta)] / n ! \\
n=0,1,2,3,4, \ldots \quad \theta>0 \quad 0 \leq \zeta \leq 1
\end{gathered}
$$

The mean and variance of this probability distribution are finite when $\zeta<1$ and are given by:

$$
E[N]=\theta(1-\zeta)^{-1} \quad \operatorname{var}(N)=\theta(1-\zeta)^{-3}
$$

When $\zeta=0$ this distribution reduces to the well-known Poisson distribution.

CONSUl and SHENTON (1972) derive (1.1) using Lagrange's expansion. A probabilistic derivation of the Lagrangian Poisson distribution can be found in GoOvaerTs and KaAs (1991). Once we know this distribution it is possible to recognize it in the literature. Browsing in COX and Miller (1965), I encountered it as a queueing exercise on page 250. They refer to MCMILlan and RiORdaN (1957).

Clearly, the Lagrangian Poisson is a useful distribution which belongs to the statistical toolkit.

A generalization of the loglinear Poisson model using the Lagrangian Poisson may be useful. This allows the variance to exceed the mean. Maximum likelihood

\footnotetext{
I This adheres to the terminology in CONSUL and SHENTON (1972). The distribution is also known as Generalized Poisson. The adjective generalized, being rather uninformative anyway, applies also to arbitrary mixed and compound Poisson distributions.
} 
analysis for a simple model is given in the next section, followed with the exposition for a loglinear model.

Evaluation of Information matrices needs the following moments, which can be found on page 157 in Consul (1989):

$$
\begin{aligned}
& E\left[\frac{N-1}{(\theta+N \zeta)^{2}}\right]=\frac{\theta(1-\zeta)+2 \zeta}{\theta(\theta+2 \zeta)}-\theta^{-2} \\
& E\left[\frac{N(N-1)}{(\theta+N \zeta)^{2}}\right]=\frac{\theta}{\theta+2 \zeta} \\
& E\left[\frac{N^{2}(N-1)}{(\theta+N \zeta)^{2}}\right]=\frac{\theta(\theta+2)}{(1-\zeta)(\theta+2 \xi)}
\end{aligned}
$$

A numerical illustration and some final remarks complete the paper.

2. A SIMPLE MODEL

The convolution of two independent Lagrangian Poisson random variables with parameters $\left(\theta_{1}, \zeta\right)$ and $\left(\theta_{2}, \zeta\right)$ is Lagrangian Poisson with parameters $\left(\theta_{1}+\theta_{2}, \zeta\right)$. This justifies the replacement of $\theta$ in (1.1) by $m \theta$, where $m$ is a known size factor. This situation may be appropriate for a statistical agency, which collects economywide data on the number of policies and number of claims. Let there be $R$ reporting units numbered $r=1, \ldots, R$. Minus the logarithm of the likelihood function can be written as:

$$
\begin{gathered}
f=c+\theta \sum m_{r}+\zeta \sum n_{r}+\sum\left(n_{r}-1\right) \ln a_{r}-R \ln \theta \\
a_{r}=\left(m_{r} \theta+n_{r} \zeta\right)^{-1} \quad c=\sum\left[\ln n_{r} !-\ln m_{r}\right]
\end{gathered}
$$

where $\sum$ denotes summation over $r$. The elements of the gradient of $f$ result as:

$$
\frac{\partial f}{\partial \theta}=\sum m_{r}-R \theta^{-1}-\sum m_{r}\left(n_{r}-1\right) a_{r}
$$

$$
\frac{\partial f}{\partial \zeta}=\sum n_{r}-\sum n_{r}\left(n_{r}-1\right) a_{r}
$$

Following page 102 in Consul (1989), we multiply (2.2) by $\theta$ and (2.3) by $\zeta$. Adding together and equating to 0 results in :

$$
\theta \sum m_{r}=(1-\zeta) \sum n_{r}
$$


So, the implied maximum likelihood estimator for the population mean is given by the sample mean. The elements of the Hessian of $f$ can be displayed in partitioned matrix form as :

$$
\mathbf{H}=\frac{\partial^{2} f}{\partial\left[\begin{array}{l}
\theta \\
\varsigma
\end{array}\right] \partial\left[\begin{array}{l}
\theta \\
\varsigma
\end{array}\right]^{\prime}}=R \theta^{-2}\left[\begin{array}{ll}
1 & 0 \\
0 & 0
\end{array}\right]+\sum\left(n_{r}-1\right) a_{r}^{2}\left[\begin{array}{l}
m_{r} \\
n_{r}
\end{array}\right]\left[\begin{array}{l}
m_{r} \\
n_{r}
\end{array}\right]^{\prime}
$$

If we have a pathological sample for which all $n_{r}=0$, the Hessian will be a zero-matrix. In case also $n_{r}=1$ are observed, the Hessian will be positive semi-definite. In all other cases the Hessian is positive definite, which implies $f$ to be convex with a unique stationary point.

Searching along the line (2.4) we derive that this stationary point will have $\zeta<1$ and $\zeta>0$ depending on the sample. To this end we substitute (2.4) in (2.1) and get a convex function in $\zeta$ :

$$
\tilde{f}=\left(c+\sum n_{r}-R \ln \hat{\lambda}\right)-R \ln (1-\zeta)-\sum\left(n_{r}-1\right) \ln \left[m_{r} \hat{\lambda}(1-\zeta)+n_{r} \zeta\right]
$$

where $\hat{\lambda}=\sum n_{r} / \sum m_{r}$, the sample mean. Numerically, $\tilde{f}$ is defined on the open interval $(z, 1)$ where $z<0$ is given as:

$$
z=1-\min _{r}\left[n_{r} u_{r}^{-1} \mid u_{r}>0\right] \quad u_{r}=n_{r}-m_{r} \hat{\lambda}
$$

At the boundaries of this interval $\tilde{f}$ approaches $+\infty$. Probabilisticaly, the stationary point of $\tilde{f}$ should be in the interval $[0,1]$. Differentiation of $(2.6)$ results in:

$$
\frac{d \tilde{f}}{d \xi}=R(1-\zeta)^{-1}-\sum\left(n_{r}-1\right) u_{r}\left(m_{r} \hat{\lambda}+\zeta u_{r}\right)^{-1}
$$

Taking the limit of $(2.7)$ for $\zeta \rightarrow 1$ results in $+\infty$. So, the search for a stationary point, starting in this limit point, will be in the direction of $\zeta<1$. Next we evaluate (2.7) for $\zeta=0$. When this value is negative, the stationary point will have $\xi>0$. This condition can be simplified to:

$$
\left(\sum n_{r}\right)^{2}<\left(\sum m_{r}\right)\left[\sum n_{r}\left(n_{r}-1\right) / m_{r}\right]
$$

When all $m_{r}$ are equal, this condition amounts to the statement that the sample variance exceeds the sample mean. This agrees with the findings on page 102 in Consul (1989).

So, there is a possibility that the stationary point will have $\zeta<0$. In such cases we should decide to use the Poisson model by setting $\zeta=0$. 
The Information matrix results as the expectation of (2.5). Using the expectations in (1.2) and adjusting for $m_{r}$ we get:

$$
\text { (2.8) } E[\mathbf{H}]=\sum m_{r}\left(m_{r} \theta+2 \zeta\right)^{-1}\left[\begin{array}{cc}
m_{r}(1-\xi)+2 \xi \theta^{-1} & m_{r} \theta \\
m_{r} \theta & (1-\zeta)^{-1} \theta\left(m_{r} \theta+2\right)
\end{array}\right]
$$

Both the inverse of (2.5) or (2.8) give an estimator for the covariance matrix of the maximum likelihood estimator.

Whenever all $m_{r}$ are equal we may normalize $m_{r}=1$ and the sample data are conveniently stored in a frequency table. Such a situation arises when considering the number of claims per policy. In Table 1 we find data from BICHSEL (1964) together with the maximum likelihood fit.

TABLE 1

LIABILITY CLAIMS PER AUTOMOBILE POLICY, SWITZERLAND 1961

\begin{tabular}{lrr}
\hline Number of claims & Number of policies & Lagrangian Poisson fit \\
\hline 0 & 103704 & 103722.2 \\
1 & 14075 & 14003.7 \\
2 & 1766 & 1838.2 \\
3 & 255 & 248.5 \\
4 & 45 & 34.6 \\
5 & 6 & 4.9 \\
6 & 2 & 0.7 \\
\hline 7 & 0 & 0.1 \\
\hline
\end{tabular}

The maximum likelihood estimates are $\hat{\theta}=0.14455$ and $\hat{\zeta}=0.06826$ with standard deviations 0.0011 and 0.0028 .

\section{A LOGLINEAR MODEL}

We model $\theta$ in the following loglinear way, which reconciles with the loglinear Poisson model as presented in TER BERG (1980):

$$
\begin{aligned}
& \theta_{r}=\lambda_{r}(1-\xi) \\
& \lambda_{r}=\exp \left[\mathbf{x}_{r}^{\prime} \boldsymbol{\beta}\right]
\end{aligned}
$$

where $\mathbf{x}_{r}$ is a vector of explanatory variables and $\boldsymbol{\beta}$ a parameter vector with $K$ elements.

Following the interpretation of the $R$ reporting units with different size given by $m_{r}$ this model is capable of incorporating differences between reporting units and differences between time periods by introducing appropriate dummies. A more common interpretation is the modelling of claim frequencies as a step towards a multiplicative rating structure.

The $R \times K$ matrix $\mathbf{X}$ is defined as :

$$
\mathbf{X}=\left[\begin{array}{llll}
\mathbf{x}_{1} & \mathbf{x}_{2} & \ldots & \mathbf{x}_{R}
\end{array}\right]^{\prime}
$$

and is assumed to have full column rank. The first column of $\mathbf{X}$ equals $\mathbf{1}$, a vector containing the elements 1 . 
Minus the logarithm of the likelihood function is a function of $\boldsymbol{\beta}$ and $\zeta$ and can be written as:

$$
\begin{gathered}
f=c+(1-\zeta) \sum m_{r} \lambda_{r}+\zeta \sum n_{r}+\sum\left(n_{r}-1\right) \ln a_{r}-R \ln (1-\zeta)-\mathbf{1}^{\prime} \mathbf{X} \boldsymbol{\beta} \\
a_{r}=\left(m_{r} \theta_{r}+n_{r} \zeta\right)^{-1}
\end{gathered}
$$

Differentiation of $f$ with respect to $\boldsymbol{\beta}$ and $\xi$ results in the elements of the gradent $\mathbf{g}$ :

$$
\begin{aligned}
& \frac{\partial f}{\partial \boldsymbol{\beta}}=(1-\zeta) \sum\left[1-\left(n_{r}-1\right) a_{r}\right] m_{r} \lambda_{r} \mathbf{x}_{r}-\mathbf{X}^{\prime} \mathbf{1} \\
& \frac{\partial f}{\partial \zeta}=R(1-\zeta)^{-1}+\sum\left[1-\left(n_{r}-1\right) a_{r}\right] u_{r} \quad u_{r}=n_{r}-m_{r} \lambda_{r}
\end{aligned}
$$

Differentiation of $\mathbf{g}$ with respect to $\boldsymbol{\beta}$ and $\zeta$ results in the elements of the Hessian $\mathbf{H}$ :

$$
\begin{aligned}
& \frac{\partial^{2} f}{\partial \boldsymbol{\beta} \partial \boldsymbol{\beta}^{\prime}}=\sum\left[1-n_{r}\left(n_{r}-1\right) a_{r}^{2} \zeta\right] m_{r} \theta_{r} \mathbf{x}_{r} \mathbf{x}_{r}^{\prime} \\
& \frac{\partial^{2} f}{\partial \boldsymbol{\beta} \partial \zeta}=-\sum\left[1-n_{r}\left(n_{r}-1\right) a_{r}^{2}\right] m_{r} \lambda_{r} \mathbf{x}_{r} \\
& \frac{\partial^{2} f}{\partial \zeta^{2}}=R(1-\zeta)^{-2}+\sum\left(n_{r}-1\right)\left[a_{r} u_{r}\right]^{2}>0
\end{aligned}
$$

The Information matrix is given as the expected value of the Hessian:

$$
\begin{aligned}
E[\mathbf{H}]=(1-\zeta) & \sum m_{r} \theta_{r}\left[\begin{array}{c}
\mathbf{x}_{r} \\
0
\end{array}\right]\left[\begin{array}{c}
\mathbf{x}_{r} \\
0
\end{array}\right]^{\prime} \\
& +2 \sum m_{r} \theta_{r}\left(m_{r} \theta_{r}+2 \zeta\right)^{-1}\left[\begin{array}{c}
\zeta \mathbf{x}_{r} \\
(\xi-1)^{-1}
\end{array}\right]\left[\begin{array}{c}
\zeta \mathbf{x}_{r} \\
(\zeta-1)^{-1}
\end{array}\right]^{\prime}
\end{aligned}
$$

The Newton-Raphson search direction is given by $\mathbf{H}^{-1}$ g. Whenever the Hessian $\mathbf{H}$ is not positive definite, we replace the Hessian by the Information matrix. Occasionally, an iteration may imply $\zeta \geq 1$, an $a_{r} \leq 0$ or an increase of $f$. In such cases, we halve the stepsize.

Taking the loglinear Poisson model as a starting value, convergence is quick and swift. 


\section{NUMERICAL ILLUSTRATION}

In Table 2 we find data from BAILEY and SIMON (1960) on $R=20$ risk groups defined by 2 risk indicators with 5 and 4 levels.

TABLE 2

Exposure AND Number of Claims, AUtomobile liability, CANADA 1957-1958

\begin{tabular}{|c|c|c|c|c|}
\hline \multirow{2}{*}{$\mathbf{X}$-matrix } & \multirow{2}{*}{$\begin{array}{c}\text { Earned car } \\
\text { years }\end{array}$} & \multicolumn{3}{|c|}{ Number of claims } \\
\hline & & Observed & Poisson & Lagrangian Poisson \\
\hline 10000000 & 2757520 & 217151 & 219950.1 & 219868.7 \\
\hline 11000000 & 130535 & 14506 & 14052.3 & 14083.6 \\
\hline 10100000 & 247424 & 31964 & 31546.8 & 31590.9 \\
\hline 10010000 & 156871 & 22884 & 21170.2 & 21085.6 \\
\hline 10001000 & 64130 & 6560 & 6345.7 & 6394.7 \\
\hline 10000100 & 130706 & 13792 & 13688.2 & 13761.4 \\
\hline 11000100 & 7233 & 1001 & 1022.3 & 1030.5 \\
\hline 10100100 & 15868 & 2695 & 2656.3 & 2675.3 \\
\hline 10010100 & 17707 & 3054 & 3137.4 & 3142.8 \\
\hline 10001100 & 4039 & 487 & 524.7 & 531.8 \\
\hline 10000010 & 163544 & 19346 & 18607.9 & 18631.6 \\
\hline 11000010 & 9726 & 1430 & 1493.5 & 1499.3 \\
\hline 10100010 & 20369 & 3546 & 3704.6 & 3715.9 \\
\hline 10010010 & 21089 & 3618 & 4059.7 & 4050.1 \\
\hline 10001010 & 4869 & 613 & 687.3 & 693.7 \\
\hline 10000001 & 273944 & 37730 & 35772.8 & 35715.3 \\
\hline 11000001 & 21504 & 3421 & 3789.9 & 3793.6 \\
\hline 10100001 & 37666 & 7565 & 7862.3 & 7863.5 \\
\hline 10010001 & 56730 & 11345 & 12533.7 & 12468.2 \\
\hline 10001001 & 8601 & 1291 & 1393.3 & 1402.4 \\
\hline
\end{tabular}

Applying the maximum likelihood model of the previous section, we have $K=$ $1+(5-1)+(4-1)=8$ elements for $\boldsymbol{\beta}$. The maximum likelihood results are presented in Table 3.

TABLE 3

MAXIMUM LIKELIHOOD ESTIMATES AND STANDARD DEVIATIONS

\begin{tabular}{|c|c|c|c|c|}
\hline \multirow{3}{*}{ Parameter } & \multirow{3}{*}{ 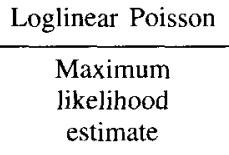 } & \multicolumn{3}{|c|}{ Loglinear Lagrangian Poisson } \\
\hline & & \multirow{2}{*}{$\begin{array}{l}\text { Maximum } \\
\text { likelihood } \\
\text { estimate }\end{array}$} & \multicolumn{2}{|c|}{ Standard deviation based on } \\
\hline & & & Hessian & Information matrix \\
\hline$\beta_{1}$ & -2.5287 & -2.5291 & .0110 & .0111 \\
\hline$\beta_{2}$ & .2998 & .3024 & .0392 & .0392 \\
\hline$\beta_{3}$ & .4691 & .4708 & .0273 & .0272 \\
\hline$\beta_{4}$ & .5259 & .5222 & .0294 & .0291 \\
\hline$\beta_{5}$ & .2156 & .2236 & .0575 & .0575 \\
\hline$\beta_{6}$ & .2723 & .2780 & .0385 & .0385 \\
\hline$\beta_{7}$ & .3552 & .3568 & .0337 & .0336 \\
\hline$\beta_{8}$ & .4930 & .4917 & .0247 & .0244 \\
\hline$\zeta$ & 0 & .8154 & .0294 & .0294 \\
\hline
\end{tabular}


We see close agreement between the parameter estimates for $\boldsymbol{\beta}$ in the loglinear Poisson as well as the loglinear Lagrangian Poisson model. This agreement is also evident in the fitted claim numbers in Table 2. The relative large value for $\zeta$ increases the variances in the Lagrangian Poisson model.

The similarity between the standard deviations based on the inverse of the Hessian and Information matrix is reassuring.

If, in this illustration, we shrink the $\mathbf{X}$-matrix to the first column, the maximum likelihood estimate for $\beta_{1}$ changes to -2.3295 and that for $\xi$ increases to 0.9738 .

\section{FINAL REMARKS}

The use of the Lagrangian Poisson distribution with loglinear mean contains, through the parameter $\zeta$, a diagnostic tool to infer the presence of omitted explanatory variables in the loglinear specification. As such, the loglinear Lagrangian Poisson model is a possible starting point from which to model the loglinear Poisson model.

When the maximum likelihood estimate for $\zeta$ is clearly different from 0 , the variance of the Poisson distribution is too small, whereas the Lagrangian Poisson distribution implies the appropriate larger variances.

As shown by GoovaerTs and KaAs (1991) the Lagrangian Poisson distribution also allows a recursive evaluation of a compound Lagrangian Poisson distribution. So, from an applied point of view, there is little reason to object to the use of the Lagrangian Poisson distribution.

\section{REFERENCES}

BAILEY, R. A. and Simon, L. J. (1960) Two studies in automobile insurance ratemaking. ASTIN Bulletin 1, 192-217.

BICHSEL, F. (1964) Erfahrungs-Tarifierung in der Motorfahrzeughaftpflicht-Versicherung. Mitteilungen der Vereinigung schweizerischer Versicherungsmathematiker 64, 119-129.

Consul, P.C. (1989) Generalized Poisson Distributions. Marcel Dekker, New York.

Consul, P.C. and SHENTON, L. R. (1972) Use of Lagrange expansion for generating discrete generalized probability distributions. SIAM Journal of Applied Mathematics 23, 239-248.

Cox. D. R. and Miller, H.D. (1965) The Theory of Stochastic Processes. Chapman \& Hal, London. GoovaERTS, M. and KAAS, R. (1991) Evaluating compound generalized Poisson distributions recursively. ASTIN Bulletin 21, 193-198.

MCMillan, B. and Riordan, J. (1957) A moving single server problem. Annals of Mathematical Statistics 28, 471-478.

TER Berg, P. (1980) On the loglinear Poisson and Gamma model. ASTIN Bulletin 11, 35-40. 